\title{
DISCLAIMER
}

\begin{abstract}
This report was prepared as an account of work sponsored by an agency of the United States Government. Neither the United States Government nor any agency thereof, nor any of their employees, makes any warranty, express or implied, or assumes any legal liability or responsiprocess disclosed, or represents thess, or usefulness of any information, apparatus, product, or ence herein to any specific ence herein to any specific commercial product, process, or service by trade name, trademark, mendation, or otherwise does not necessarily constitute or imply its endorsement, recommendation, or favoring by the United States Government or any agency thereof. The views United States Government expressed herein do not necessarily state or reflect those of the
\end{abstract}

\section{Precision Electromagnetic Calorimetry with Liquid Krypton}

\author{
Helio Takai ${ }^{\mathrm{a}}$ \\ aPhysics Department, \\ Brookhaven National Laboratory, \\ Upton, NY 11973 - USA
}

Test beam results of a liquid krypton electromagnetic calorimeter with projective accordion electrode are presented. The electrode design includes a fine segmentation section to enhance the $\pi^{0}$ rejection and pointing. The test was carried out at the H4 beam line at the CERN SPS with electron beams of energy from 20 to 200 $\mathrm{GeV}$. Preliminary results of energy resolution, linearity, $\mu$ response are presented. We also present the dependence of the energy resolution on the amount of inactive material in front of the calorimeter.

\section{Introduction}

The physics requirements of the LHC demands the development of a fast, stable, finely segmented calorimeter with excellent energy resolution and a small constant term at high energies. Experiments designed for LHC[1] and SSC[2] to search for the Higgs decay into two photons, $H^{0} \rightarrow \gamma \gamma$, require very good energy resolution and background rejection. The aim of this test has been: a) to bring the energy resolution of an EM accordion calorimeter[3,4] into the range of about $5.5 \% / \sqrt{E}$ with a small constant term; b) to achieve good position and pointing resolution by using a finely segmented first section that will act as an integrated pre-shower detector[2]; and c) to study the energy resolution dependence on the amount of inactive material in front of the calorimeter.

To improve the energy resolution we have chosen to increase the sampling fraction by replacing the liquid argon (LAr) with the next heavier noble liquid, liquid krypton ( $L K r)$. Thus for the same calorimeter one achieves a higher sampling frequency and a higher sampling fraction. A non projective accordion has been tested by us at BNL[5-8]. In this test we have built a projective accordion module with an integrated pre-shower section.

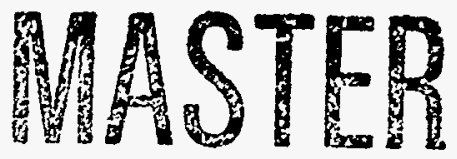

\section{Experimetal setup}

The test of the calorimeter was conducted at the $\mathrm{H} 4$ beam line at the SPS at CERN, in which negative beams of electrons, in the momentum range from 20 to $200 \mathrm{GeV} / \mathrm{c}$, as well as pions and muons are available. The calorimeter test setup is schematically shown in Fig. 1. Two scintillation counters $\mathrm{S} 1$ and $\mathrm{S} 2$ of dimensions $2.5 \times 2.5 \mathrm{~cm}^{2}$ and $3 \times 3 \mathrm{~cm}^{2}$ respectively were used to define the beam direction and time $t_{0}$ for the test run. In front of the calorimeter a set of four veto scintillators covered with $6 \mathrm{~mm}$ thick $\mathrm{Pb}$ plates were placed in order to reject those events in which the shower process starts upstream of the calorimeter. The veto counters define a beam spot size of $2 \times 2 \mathrm{~cm}^{2}$ for the calorimeter. Two Cathode Strip Chambers[9] (CSC) were used to determine the incident particle position on the face of the calorimeter. The CSC position resolution are nominally better then $70 \mu \mathrm{m}$.

\subsection{The Calorimeter Module}

The shape of the test calorimeter is a projective cylindrical wedge with a front face of dimensions $60 \times 37.5 \mathrm{~cm}^{2}$ and depth of $55 \mathrm{~cm}$ which corresponds to $25 X_{0}$ with liquid krypton. An isometric view of the accordion showing the electrical connections, and internal electronics is shown in Fig. 2. The absorber plates are made of $1.0 \mathrm{~mm}$ $\mathrm{Pb}$ plates sandwiched between two sheets of 0.2 $\mathrm{mm}$ stainless steel. Stainless steel provides both 


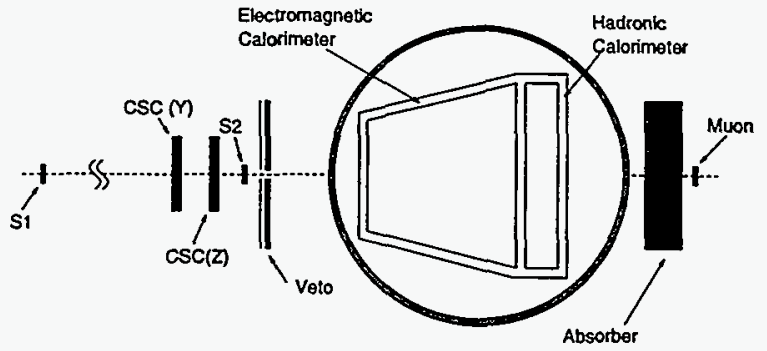

Figure 1. Experimental setup for the test run.

the mechanical strength as well as a better surface than lead. There is a nominal $2 \mathrm{~mm}$ liquid gap between the absorber and the electrode. A small hadronic module of an EST parallel plate design followed the electromagnetic module.

\subsection{Readout Electronics}

The readout chain used in the experiment is show in Fig. 3. Both the pre-amplifier and the calibration circuits are located as close as possible to the signal electrodes and are operated at cryogenic temperatures [11]. The high voltage connections to the electrodes are distributed over busses located on the electrode boards. The motherboard contains routing for all output signals, calibration signals, and low voltage power. Transmission lines of $50 \Omega$ characteristic impedance terminated at both ends connect the preamplifier output to an intermediate amplifier and cable driver located on the exterior wall of the cryostat. The intermediate amplifier has a switchable voltage gain of 2 or 9 (the gain is fixed at 9 for the lower dynamic range front (strip) section and for the hadronic section), and drives differentially a $100 \Omega$ shielded twisted pair cable 35 meters long. The signals are received in the counting room by Variable Gain Amplifiers (VGA). The signals arriving at the VGA are split into two. One path takes the signal to a $40 \mathrm{~ns}$, bipolar shaper/amplifier and the other path is input to a current sum. The most important feature of the VGA is the adjustment of each individual channel gain within a range of $\pm 20 \%$ through a voltage-controlled capacitance in the amplifier.
The voltage is set through a 12-bit DAC driven by a on-board microprocessor. This feature of the VGA, in combination with the calibration system discussed below, permits equalization of gains to a precision of a $0.2 \%$. Thus sums within this precision can be generated. All shaped signals are fed into track and hold units and digitized by a 12-bit fastbus ADC.

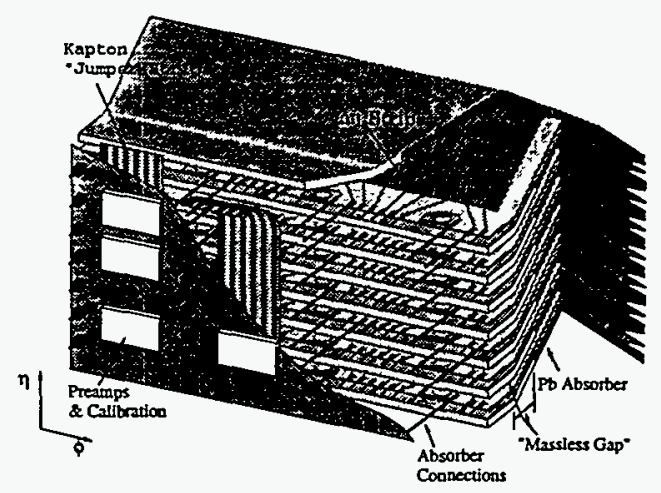

Figure 2. Isometric view of the test module. The integrated preradiator and the associated electronics is shown.

A non-loading, high input impedance circuit ("High Impedance Pick-of") branches off some of the individual channels which together with the analog sums are sampled every $25 \mathrm{~ns}$ and stored in a switched capacitor array (SCA), thus simulating the mode of data acquisition likely to be employed at the LHC. A total of 32 samples per channel were kept for offline analysis. The SCA are described in detail in ref. [10].

One of the important advantages of liquid ionization calorimetry is stability and uniformity of response. In this test we used a calibration system developed at BNL which permits the formation of the calibration pulse right at the preamplifier input: A clock signal with a rise time of 3 ns is distributed on a $50 \Omega$ transmission line embedded in the motherboard." Each channel can be 


\section{DISCLAIMER}

Portions of this document may be illegible in electronic image products. Images are produced from the best available original document. 
selected externally by means of DC level and the amount of charge injected is given by a DC current source as shown in Fig. 3. The signal shape is nearly exact to the calorimeter current signal for the corresponding shaping time. The calibration permits to address each channel and to inject a precise amount of charge. This technique has permitted an interchannel gain equalization to better than $0.2 \%$.

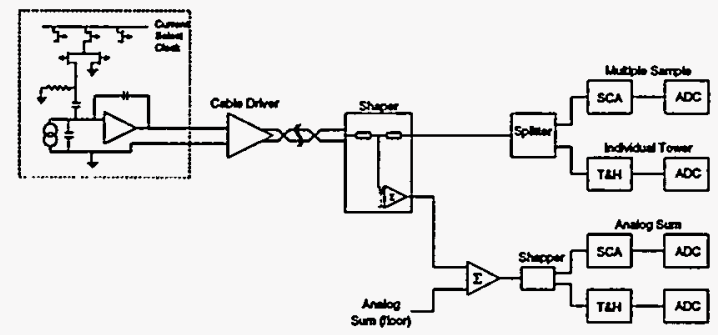

Figure 3. Electronics chain for the readout and calibration of the test module.

\subsection{Cryogenics and Purification System}

A specially made bathtub surrounded the calorimeter modules with the goal of minimizing the amount of krypton required. The cryostat is the same as was used in our previous test in 1992[6]. The krypton was cooled and liquified using a liquid nitrogen heat exchanger at the top of the cryostat.

We used the same krypton as in the 1992 test of the non projective module. However, for this run it was purified by passing it through Oxysorb filters. During the run the purity of the liquid krypton and the electron lifetime in the liquid were determined by means of a small ionization chamber and a ${ }^{241} \mathrm{Am}$ source (alpha cell) immersed in bathtub. Fig. 4a shows the response of this alpha cell as well as a curve resulting from the calculation of a convolution of the electronics impulse response with the expected krypton current signal. From the calculation a drift velocity of $3.8 \mathrm{~mm} / \mu \mathrm{s}$ at the nominal voltage of $10 \mathrm{KV}$ was derived. The total charge that the source deposit in the alpha

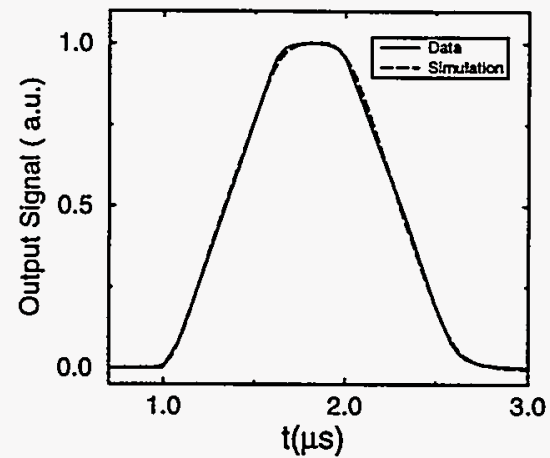

Figure 4. Current signal from the alpha cell. The solid line is the data and the dashed line is the calculated value.

cell was measured several times each day during our two week run, no measurable change in the charge during this time was found to the $1 \%$ level. We emphasize that no special treatment of the materials that entered the cryostat other than alcohol cleaning was needed and there was no need to purify the krypton once it was liquified.

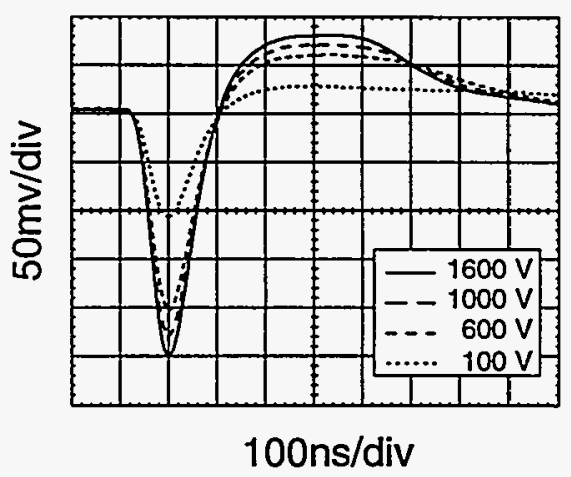

Figure 5. Online signal waveforms from the calorimeter with different voltage settings 


\section{Experimental Results}

\subsection{Calorimeter Response}

Fig. 4b displays the shaped signals from the calorimeter taken at different high voltage settings. As the applied high voltage is raised, the electron signal reaches a limiting value. This can be seen both in the pulse shape and in amplitude. In liquid krypton, however, the drift velocity is already large at very low voltages and even at $50 \mathrm{~V}$ a pulse with $30 \%$ of the maximum amplitude can be seen. The data we are reporting in this paper were taken at $2000 \mathrm{~V}$.

\subsection{Energy Resolution}

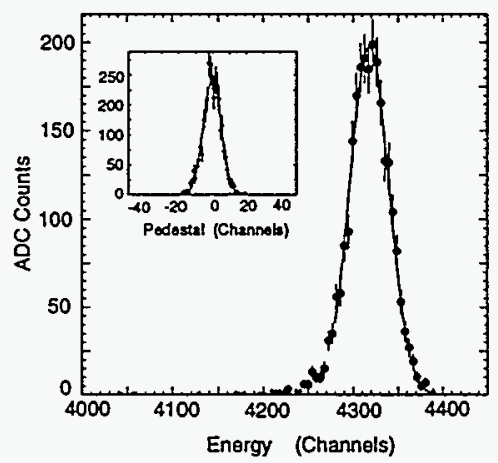

Figure 6. Energy spectrum for $200 \mathrm{GeV}$ incident electrons for the total energy sum.

To determine the energy resolution of the calorimeter, we define an active region to be a $6 \times 6$ tower configuration around the position of the incident particle. The energy was corrected for $\phi$ modulation, energy loss in the front of the calorimeter as well as energy leakage at the back. Minimal corrections are needed to compensate for the amount of energy deposited before the active part of the calorimeter, due to the presence of a passive massless gap. Using the corrected data, we show in Fig. 6a the energy spectrum obtained for $200 \mathrm{GeV}$ electrons. The distribution of pedestal events are shown in the figure, and their width obtained from Gaussian fits are $\sigma=240 \mathrm{MeV}$ for 30 towers. Thus the equivalent noise for a single readout tower is $43 \mathrm{MeV}$ for the low-low gain setting. The rms value divided by the mean of the spectrum in Fig. 6a energy resolution of $5.5 \% / \sqrt{E}$, after the noise contribution is subtracted in quadrature. There is no correction for the momentum spread of the beam.

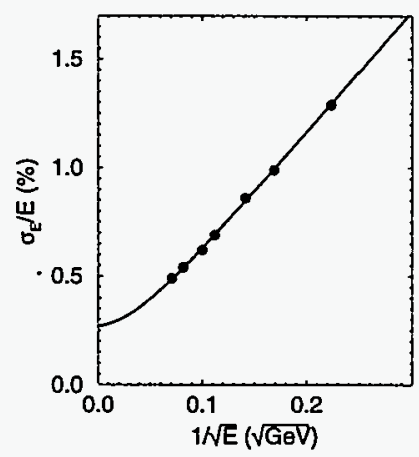

Figure 7. Energy resolution and function of the incident electron energy. Error bars are smaller than the points in the graph.

The fitting procedure was repeated for each of the eight beam momenta at which data were taken from 20 to $200 \mathrm{GeV} /$ c. In Fig. 6 b, we show these data, along with a line fitted to the function

$$
\frac{\sigma_{E}}{E}=a \oplus \frac{b}{\sqrt{E}} \text {. }
$$

The fitted values of the parameters are $a=$ $(0.27 \pm 0.2) \%$ and $(b=0.055 \pm 0.004) \sqrt{\mathrm{GeV}}$.

The linearity of the calorimeter response, over a range from $20 \mathrm{GeV}$ to $200 \mathrm{GeV}$, is defined as the ratio of the fitted mean energy to the nominal beam energy. The relative deviations from a straight line as a function of beam momentum have been measured and the system is found to be linear over this energy range to within $0.1 \%$.

\subsection{Energy resolution as a function of in- active material}

In a realistic electromagnetic calorimeter for a collider experiment, inactive material in front of 


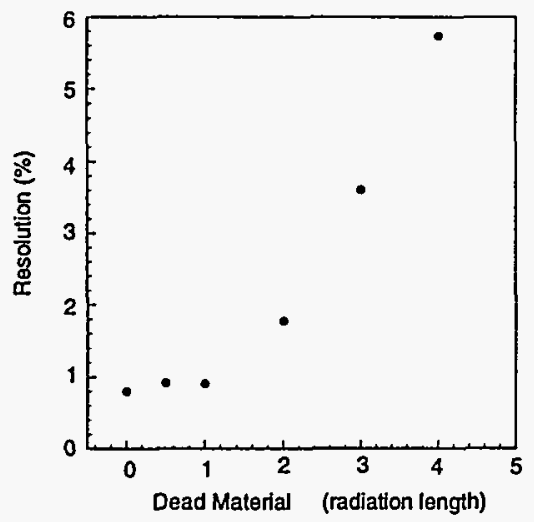

Figure 8. Energy deposited in the calorimeter as a function of the additional amount of material in front of the calorimeter.

the active calorimeter is inevitable. In this test, the amount of inactive material in front of the active calorimeter is $1.2 \mathrm{X}_{0}$ in the regular setup. To study the calorimeter response to additional inactive material, lead plates of various thicknesses were placed immediately upstream of the cryostat. The total amount of inactive material varied from $1.2 X_{0}$ to $5.2 X_{0}$.

A $50 \mathrm{GeV}$ electron beam was used in this study. The total observed energy is plotted as a function of total inactive material in Fig. 8. The resolution is determined from energy distributions where weights for each longitudinal sections were optimized for each case. It is clear that the addition of inactive material of $2.0 X_{0}$ thickness results in a non-recoverable energy resolution.

\subsection{Position and Pointing resolution}

The shower position is measured with the transverse segmentation of the calorimeter for each longitudinal section. In the front section, three strips centered around the one with highest energy are used in the position calculation, while in the towers, $3 \times 3$ towers centered around the tower with highest energy are used. Fits to the curves are then used to correct for the non-linear relation between the impact point and simple en- ergy weighted position. The position resolution is the width of the difference between the chamber position and position measured by the calorimeter. The noise contribution to the position measurement is negligible at the energies under study.

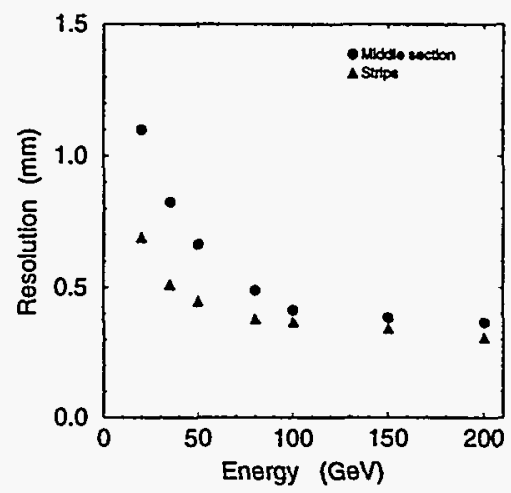

Figure 9. Position resolution for the Strip section and Middle section.

Fig. $8 \mathrm{~b}$ shows the position resolution as a function of the beam momentum for the strips and middle longitudinal segment. A fit of the form

$$
\sigma=a / \sqrt{E} \oplus b
$$

gives the parameters $a=2.7 \mathrm{~mm} \mathrm{GeV} V^{1 / 2}, b=0.24$ $\mathrm{mm}$ for the strips, and $\mathrm{a}=4.4 \mathrm{~mm} \mathrm{GeV} / 12$ and $\mathrm{b}=$ $0.3 \mathrm{~mm}$ for the middle longitudinal segment.

Using the positions measured at the three different depths, the angle of the calorimeter shower is also measured. In this calculation the average shower centroid in the longitudinal direction for each longitudinal segment is assumed according to the Monte Carlo simulation. The $\theta$ angular resolution obtained can be parameterized as

$$
\sigma_{\theta}=(41 \pm 0.4 / \sqrt{E} \oplus 2.0) \mathrm{mrad}
$$

\subsection{Response to Muons}

The calorimeter muon response is displayed in Fig. 10. The muon energy was defined as the 
energy deposited in a $2 \times 1$ tower geometry. The pedestal (in units of energy) is displayed along with the data and illustrates the signal to noise one can achieve with this system.

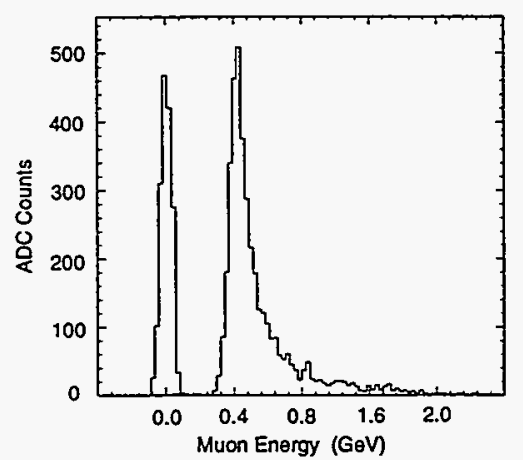

Figure 10. Muon Energy deposition for the High High configuration.

The suppression of the response of an electromagnetic shower relative to that of a minimum ionizing particle is usually called the $e / \mu$ ratio, which we define as the ratio of the electron response to the muon response (peak) for a given amount of energy lost in the calorimeter. The muon total energy loss was computed using the GEANT simulation program. Using the linear response curve obtained in the electron runs, the electron response for the same amount of energy loss was calculated. The $e / \mu$ ratios for liquid krypton is $0.83 \pm 0.01$.

\subsection{Timing Resolution}

The timing performance of the calorimeter was measured by discriminating the shaped signal from the middle section analog sum. The discriminator used has been described earlier [5]. The timing was measured in a TDC which was started by the beam scintillators and stopped by the output of the constant fraction discriminator (CFD). The online information is corrected offline for difference in cable lengths using the algorithm of Ref [5]. The timing resolution, shown in Fig 11 achieved during the test for electrons of $120 \mathrm{GeV}$

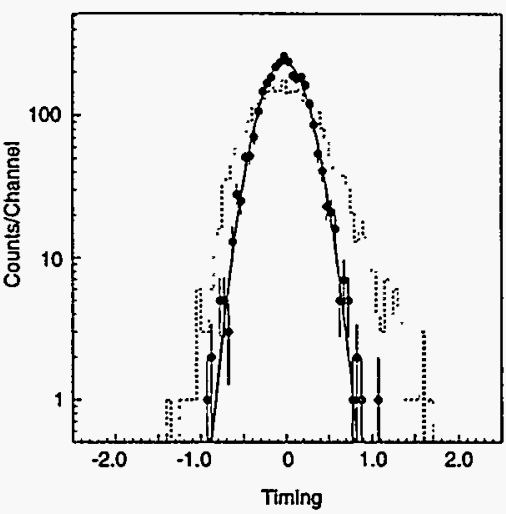

Figure 11. Timing response of the calorimeter for electrons of $120 \mathrm{GeV}$.

is 260 ps. This resolution was limited by the scintillator timing resolution of $190 \mathrm{ps}$ and largely by the shaper (second stage) noise.

\subsection{Photon Data}

To study the calorimeter response to photons the experimental setup presented in section 2 was modified to allow the tagging of photons produced by a target. This is illustrated in Fig. 12. The calorimeter now detects both electrons and photons produced upstream of a dipole magnet which deflects the electrons. To reduce the number of events with multiple photon a veto counter with $\mathrm{a} \mathrm{Pb}$ converter is placed in front of the calorimeter.

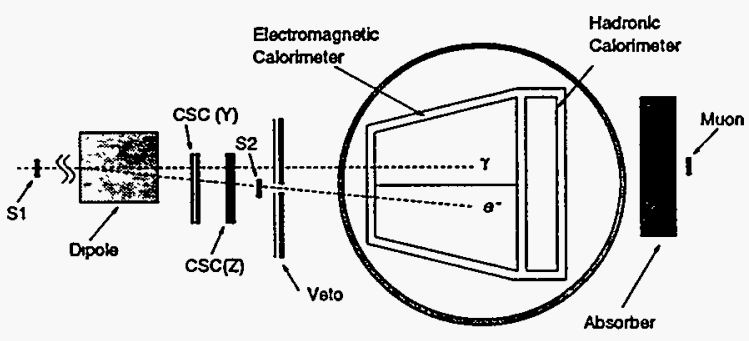

Figure 12. Experimental setup for the photon response measurements. 
With this setup photons of energy between 3580 were detected. The analysis of the data is ongoing, in Fig. 10b we show a typical event dump where one can immediately see the advantages of the integrated preshower for $\gamma / \pi^{0}$ separation. The photon signal in the preshower is narrow and well separated from the noise. This will enable us to do a detailed shower shape analysis. General features of the data are in good agreement with the Monte Carlo but detailed analysis and comparison is now ongoing.

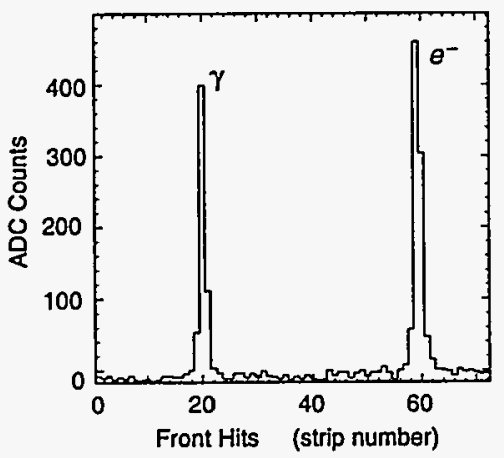

Figure 13. Event dump showing the energy profile of a photon and the electron from the tagged beam

\section{Discussion and Conclusion}

We have tested successfully a projective liquid krypton accordion calorimeter with an integrated preshower. We found: i) The krypton signal was stable over the two weeks of the run, with no purification needed. ii) The energy resolution obtained was in good agreement with the MC expectation, showing that the accordion design can achieve resolution below $6 \%$ with a very small constant term. iii) Pointing resolution of the order of $40 \mathrm{mrad} / \sqrt{E}$ can be obtained with an integrated preshower. iv) Preliminary result on the shower shape analysis of photons look very encouraging. v) Inactive material does not deteriorate the calorimeter response significantly below
$2 \mathrm{X}_{0}$. vi) The passive massless gap in the absorber minimizes the need for correction as a function of longitudinal shower development.

\section{Acknowledgments}

Our design of the accordion electrode structure has relied on the pioneering work of the RD3 collaboration. We would like to thank the many people at CERN who were more than helpful during the test. In particular we would like to thank the teams from RD3/ATLAS, NA48 who have given us special help during the run. We also acknowledge the loan of the krypton from the Budker Institute for Nuclear Physics.

This research was supported in part by the U.S. Department of Energy under contract DE-AC02$76 \mathrm{CH} 00016$. Part of this work is supported by the SSC closeout funds.

\section{References}

\section{REFERENCES}

1. ATLAS, Conceptual design report

2. GEM experiment Technical Design Report, GEM TN-93-262 (1993) unpublished.

3. B. Aubert et al., Nucl. Inst. and Methods A309 (1991) 438.

4. B. Aubert et al., Nucl. Inst. and Methods A321(1992) 467.

5. O. Benary et al., Nucl. Inst. and Methods, A344,(1994)78.

6. O. Benary et al., Nucl. Instr. and Methods, A344(1994)363

7. O. Benary et al., Nucl. Instr. and Methods, A350(1994)131

8. O. Benary et al., Nucl. Instr. and Methods, A349(1994)367

9. G. Bencze et al., BNL Report 60837, (1994) Nucl. Instr. and Methods (in Press)

10. J. Parsons, Proceedings of the V International Conference on Calorimetry for High Energy Physics, Brookhaven, September,25 - October, 1 - 1994.

11. V. Radeka and S. Rescia, Nucl. Inst. and Methods $\underline{\text { A265 }}$ (1988) 228 